\title{
Variation in human mobility and its impact on the risk of future COVID-19 outbreaks in Taiwan
}

\author{
Meng-Chun Chang ${ }^{1 \dagger}$, Rebecca Kahnn ${ }^{2 \dagger}$, Yu-An Li ${ }^{1}$, Cheng-Sheng Lee ${ }^{3}$, Caroline O. Buckee ${ }^{2}$ and \\ Hsiao-Han Chang ${ }^{1 *}$ (ID
}

\begin{abstract}
Background: As COVID-19 continues to spread around the world, understanding how patterns of human mobility and connectivity affect outbreak dynamics, especially before outbreaks establish locally, is critical for informing response efforts. In Taiwan, most cases to date were imported or linked to imported cases.

Methods: In collaboration with Facebook Data for Good, we characterized changes in movement patterns in Taiwan since February 2020, and built metapopulation models that incorporate human movement data to identify the high risk areas of disease spread and assess the potential effects of local travel restrictions in Taiwan.

Results: We found that mobility changed with the number of local cases in Taiwan in the past few months. For each city, we identified the most highly connected areas that may serve as sources of importation during an outbreak. We showed that the risk of an outbreak in Taiwan is enhanced if initial infections occur around holidays. Intracity travel reductions have a higher impact on the risk of an outbreak than intercity travel reductions, while intercity travel reductions can narrow the scope of the outbreak and help target resources. The timing, duration, and level of travel reduction together determine the impact of travel reductions on the number of infections, and multiple combinations of these can result in similar impact.

Conclusions: To prepare for the potential spread within Taiwan, we utilized Facebook's aggregated and anonymized movement and colocation data to identify cities with higher risk of infection and regional importation. We developed an interactive application that allows users to vary inputs and assumptions and shows the spatial spread of the disease and the impact of intercity and intracity travel reduction under different initial conditions. Our results can be used readily if local transmission occurs in Taiwan after relaxation of border control, providing important insights into future disease surveillance and policies for travel restrictions.
\end{abstract}

Keywords: COVID-19, Taiwan, Metapopulation model, Mobility data, Travel restrictions

\section{Background}

The Coronavirus Disease 2019 (COVID-19) was first reported in Wuhan, China in December 2019 and has since caused a global pandemic, with over 15,000,000 confirmed cases and over 600,000 deaths reported by July 23,2020

\footnotetext{
* Correspondence: hhchang@life.nthu.edu.tw

${ }^{\dagger}$ Meng-Chun Chang and Rebecca Kahn are shared first authorship.

'Department of Life Science \& Institute of Bioinformatics and Structural Biology, National Tsing Hua University, Hsinchu, Taiwan

Full list of author information is available at the end of the article
}

[1]. Scientific discoveries have advanced at an unprecedented pace, with numerous clinical trials of drugs and vaccines underway $[2,3]$. In the meantime, public health officials must rely on other interventions, such as social distancing and travel restrictions, to slow the spread and reduce the peak of the outbreak, in order to prevent health systems from being overwhelmed $[4,5]$.

In January 2020, as the epidemic in Wuhan grew, many countries implemented travel bans, and airlines canceled flights to attempt to slow the spread [6]. A

(c) The Author(s). 2021 Open Access This article is licensed under a Creative Commons Attribution 4.0 International License, which permits use, sharing, adaptation, distribution and reproduction in any medium or format, as long as you give appropriate credit to the original author(s) and the source, provide a link to the Creative Commons licence, and indicate if changes were made. The images or other third party material in this article are included in the article's Creative Commons licence, unless indicated otherwise in a credit line to the material. If material is not included in the article's Creative Commons licence and your intended use is not permitted by statutory regulation or exceeds the permitted use, you will need to obtain permission directly from the copyright holder. To view a copy of this licence, visit http://creativecommons.org/licenses/by/4.0/ The Creative Commons Public Domain Dedication waiver (http://creativecommons.org/publicdomain/zero/1.0/) applies to the data made available in this article, unless otherwise stated in a credit line to the data. 
number of studies have estimated the risk of importation globally, with some suggesting up to two-thirds of all imported cases went undetected [7, 8]. For Taiwan, there have been 411 reported cases as of June 30, 2020 [9], with 356 imported (87\%) and 55 local cases (13\%). Fiftytwo local cases (94.5\%) were linked to imported or known cases, and 3 local cases (5.5\%) have unknown origin. Since February 7th, Taiwan has implemented entry restrictions on foreign nationals based on their travel histories; 14-day home quarantine started being required for visitors from certain locations from February 10th and became required for all travelers from March 19th [10]. While COVID-19 transmission in Taiwan is relatively well-controlled, the number of cases globally is still increasing [1]. When border control is relaxed in Taiwan in the future, importation from other countries has the potential to lead to local outbreaks, especially if other non-pharmaceutical interventions, such as hand washing or mask wearing, are not adopted at the same level as in March and April 2020.

As the number of cases globally due to community transmission grows relative to the number of imported cases, attention has turned to more local measures to decrease spread, such as cancellations of mass gatherings, business closures, and local travel restrictions [11]. Mobility data can provide critical information for responding to outbreaks and understanding the impact of travel restrictions [12]. Recent studies have analyzed the effects of human mobility and travel restrictions on disease spread during the COVID-19 pandemic $[6,13-$ 16]. Here, to prepare for COVID-19 and its impact, in collaboration with Facebook Data for Good, we describe the metapopulation models we've built that include human movement data to better understand the high risk areas of disease spread and assess the potential impact of local travel restrictions in Taiwan.

\section{Methods}

\section{Mobility data and geographic unit}

Mobility data can provide important insights into how people move and how these patterns change during crisis situations [17, 18]. We incorporated two different sources of mobility data from Facebook into our models: Facebook colocation data and Facebook movement data. Facebook users who have location services enabled on their smart phones contribute data to these data sets, with users' locations categorized into Bing Tiles [17, 18]. Facebook's newly developed colocation matrices (Facebook colocation data) give the probability that people from two different geographic units will be in the same $600 \mathrm{~m} \times 600 \mathrm{~m}$ location for 5 minutes using data over the course of a week. Facebook's regular movement data (Facebook movement data) aggregates the number of trips
Facebook users make between locations every 8 hours (Figure S1) [18]. Mobility data between January 26th and June 30th were used. Facebook movement data were disaggregated by weekdays (Monday to Friday), weekends, and holidays (Lunar New Year, Ching Ming festival, and Dragon Boat Festival). Facebook colocation data included weeks containing holidays and weeks containing only regular (i.e. non-holiday) days.

The geographic unit used in this study was at the centrally-governed level of "city" (here "city" indicates city, county or special municipality in Taiwan). Shape files were downloaded from Government open data platform (https://data.gov.tw/dataset/7442). We excluded three cities outside of the main island of Taiwan from the analysis due to their low connectivity with the main island, leaving 19 cities.

\section{Metapopulation models}

We developed susceptible-latent-infectious-recovered (SLIR) models of the spread of COVID-19 throughout Taiwan. In order to understand the initial stages of disease spread, we ran the models stochastically using a continuous-time Markov chain process [19]. The analogous deterministic formulation, which contains transition probabilities used in stochastic simulations, is described below. We ran the model until either (1) it reached $n$ cumulative infections or (2) the total number of infections became 0 and repeated simulations 1000 times to estimate the probability of having more than $n$ infections (denoted by $P_{n, k}$, where $k$ represents the number of initial infections), the time it takes to reach $n$ infections (denoted by $T_{n, k}$ ), and the standard deviation of infection numbers at $T_{n, k}$ (denoted by $V_{n, k}$ ). To assess the initial stages of the outbreak, we used $n=1000$ and $k=3$ as our baseline values.

Let $S_{i}, L_{i}, I_{i}, R_{i}$ be the number of susceptible, latent, infectious, and recovered individuals in location $i$, respectively, and $N_{i}$ be the total population in location $i$. Let $D_{L}$ $(=3.5)$ be the latent period, and $D_{I}(=3)$ be the duration of infectiousness [13]. Because transmission rates can change with non-pharmaceutical interventions, as shown in previous studies, we vary.

$R_{O}$ in our model $\left(R_{O}=2.4,1.2\right.$, or 0.9$)[13,20,21]$.

We modified spatial models from previous studies [22-25] and constructed two metapopulation models, a contact model and a residence model, with the former using Facebook colocation data and the latter using Facebook movement data. In the "contact model", we assumed that contact rates (and therefore transmission rates) varied among locations and was proportional to colocation probabilities $\left(C_{i j}\right.$, the probability that a person from location $i$ collocates with a person from location $j$ ) from Facebook colocation data. We scaled $R_{O}$ by $C_{i j}{ }^{*} N_{j}$ 
(for $j$ not equal to $i$ ) or $C_{i i}{ }^{*}\left(N_{i}-1\right)$, standardized to the average $C_{i i} *\left(N_{i}-1\right)$ across all 19 cities (denoted by $\left.C_{i i}\left(\bar{N}_{i}-1\right)\right)$. In rare cases where $R_{0 i i}$ was above 3.5 , we set it to a maximum value of 3.5 .

$$
\begin{aligned}
\frac{d S_{i}}{d t} & =-\sum_{j \text { include } i} S_{i} \frac{I_{j}}{N_{j}} \frac{R_{0 i j}}{D_{I}} \\
R_{0 i j} & =R_{0} \frac{C_{i j} N_{j}}{C_{i i}\left(\bar{N}_{i}-1\right)} \\
R_{0 i i} & =R_{0} \frac{C_{i i}\left(N_{i}-1\right)}{C_{i i}\left(\bar{N}_{i}-1\right)}
\end{aligned}
$$

In the "residence model", we first estimated the proportion of time people living in location $i$ spend in location $j\left(P_{i j}\right)$ based on Facebook movement data (see details in Supplementary Text), and modeled the transmission dynamics by considering both that (1) nontravelers get infected by infectious visitors to their home location (the first part in the following equation) and that (2) susceptible travelers get infected when they travel (the second part in the following equation).

$$
\frac{d S_{i}}{d t}=-S_{i} P_{i i} \frac{R_{0}}{D_{I}} \frac{\sum_{j \text { includes } i} I_{j} P_{j i}}{\sum_{j \text { includes } i} N_{j} P_{j i}}-S_{i} \sum_{j \neq i} P_{i j} \frac{I_{j}}{N_{j}} \frac{R_{0}}{D_{I}}
$$

Because the difference between residence models with and without considering the interaction occurring between visitors from different cities inside another third city was minimal (see Supplementary Text for details), for simplicity, we considered the model without the interaction occurring between visitors from different cities inside another third city. The remaining equations are the same across the two models.

$$
\begin{aligned}
& \frac{d L_{i}}{d t}=\frac{-d S_{i}}{d t}-\frac{L_{i}}{D_{L}} \\
& \frac{d I_{i}}{d t}=\frac{L_{i}}{D_{L}}-\frac{I_{i}}{D_{I}} \\
& \frac{d R_{i}}{d t}=\frac{I_{i}}{D_{I}} \\
& N_{i}=S_{i}+L_{i}+I_{i}+R_{i}
\end{aligned}
$$

In addition to using different movement data, the major difference between two models is that the transmission rate within each city $\left(R_{0} / D_{I}\right)$ varies with colocation matrices in the contact model, while it remains constant in the residence model. In this sense, the contact model is similar to the traditional density dependent model, where contact rates (and therefore transmission rates) vary with population density, and the residence model is similar to the frequency dependent model [26]. As it is unclear which is most appropriate for COVID-19, we used both and compared the results.

\section{Risk of infection and regional importation}

We defined three connectivity measures relevant for disease transmission, risk of infection, risk of regional importation, and source of importation. Using Facebook colocation data, we defined $R_{O i i}$ as intracity $R_{O}$ and $\sum_{j \neq i}$ $R_{0 i j}$ as intercity $R_{O}$ for location $i$. The sum of intracity $R_{O}$ and intercity $R_{O}$ reflects total risk of infection and was standardized to the highest value.

Risk of infection for location $i=\sum_{j \text { include } i} R_{0 i j}$.

Similarly, using Facebook movement data, we defined $\left(\sum_{j \neq i} \frac{m_{j i} N_{j}}{q_{j}}\right) / N_{i}$ as risk of regional importation (i.e. importation from other cities within Taiwan) for location $i$, where $q_{j}$ represents the average number of subscribers in location $j$ and $m_{j i}$ represents the average number of people moving from location $j$ to location $i$ per unit of time in Facebook movement data. Source of importation was defined as the number of travelers from each location $i$ and standardized to the highest value.

Source of importation for location $i=\sum_{j \neq i} \frac{m_{i j} N_{i}}{q_{i}}$.

Facebook colocation data from regular days were used to calculate risk of infection, and weekday movement data were used to calculate risk of regional importation and source of importation.

\section{Modeling travel reduction}

To assess the potential effect of travel restrictions at multiple levels, we modeled either intra-city travel reductions, inter-city travel reductions, or a combination of both travel reductions ("overall reduction" in texts and figures) for 1, 2, 3, or 6 months or for the whole period of time. Intracity travel reductions represent decreased contacts between people within the same city, which could be achieved through a combination of measures, such as individual quarantines or social distancing policies. Travel reductions started from the beginning of the simulations or when there were $10,20,30,50$, or 100 accumulated infections. The proportion of reduction is denoted by $G$. In the contact model, intracity reduction was modeled by $R_{O i i}{ }^{*}(1-G)$ for all $i$, and intercity reduction was modeled by $R_{O i j}{ }^{*}(1-G)$ for all $i$ not equal to $j$. In the residence model, intracity reduction was modeled by $\mathrm{R}_{0}{ }^{*}(1-G)$ and intercity reduction was modeled by 
$P_{i j}^{* *}(1-G)$ for all $i$ not equal to $j$ and $P_{i i}+\left(1-P_{i i}\right)^{* *} G$ for all $i$.

\section{Results}

Varying human mobility across space and time in Taiwan

We quantified how intercity and intracity mobility varied at the centrally-governed level in the past 5 months using Facebook mobility data. On average, intracity movement was 7-fold of intercity movement, and intracity colocation probability was over 200 -fold of intercity colocation probability. We quantified the difference in connectivity between locations by three measures: risk of infection, risk of regional importation, and source of importation (Fig. 1 and Table S1). Risk of infection identifies locations with larger colocation probabilities. If assuming contact rates were proportional to colocation probabilities, disease transmission rates are expected to be higher in locations with higher risk of infection, such as Taipei City, New Taipei City, and Kaohsiung City. Risk of regional importation represents the relative number of travelers and local people, and higher values indicate higher possibility that travelers will transmit virus to local people. Source of importation calculates how much travelers from each location contribute to other locations. Viruses are expected to spread more quickly if initial local infections in Taiwan occur in locations with higher values of source of infection. Taipei and New Taipei City are cities with the highest risk of regional importation and source of importation, respectively. These three measures quantify different but related aspects of mobility and connectivity that are relevant for disease transmission, and while well-connected cities tend to have high values for all three measures, there are still some differences among them.

We found intercity mobility between some of the locations first decreased and then increased in the past few months, which is consistent with the changes in the number of local cases in Taiwan and global number of cases (Figures S2 and S3), indicating the level of change that can happen without travel restrictions imposed by the government. We also observed significant changes during holidays. Lunar New Year was within the early stages of the SARS-CoV-2 outbreak, and for most of cities pairs (95\%), colocation probabilities during Lunar New Year was significantly higher than regular days, as expected during holidays. However, the proportion of city pairs with higher than usual intercity connectivity during the Ching Ming Festival, which occurred at the time when the number of cases was increasing dramatically globally and the number of local cases was just starting to decrease, decreased to $67 \%$. Dragon Boat Festival was at the time the number of local cases remained zero for more than a month, and the proportion of city pairs with higher than usual intercity mobility increased to $76 \%$.

\section{The impact of the location of initial infections on the risk of the spread}

At the end of June 2020, most cases in Taiwan were imported or linked to imported cases. Therefore, we used meta-population models parameterized by human mobility data from Facebook to simulate the spread of SARS-CoV-2 under a variety of initial conditions,

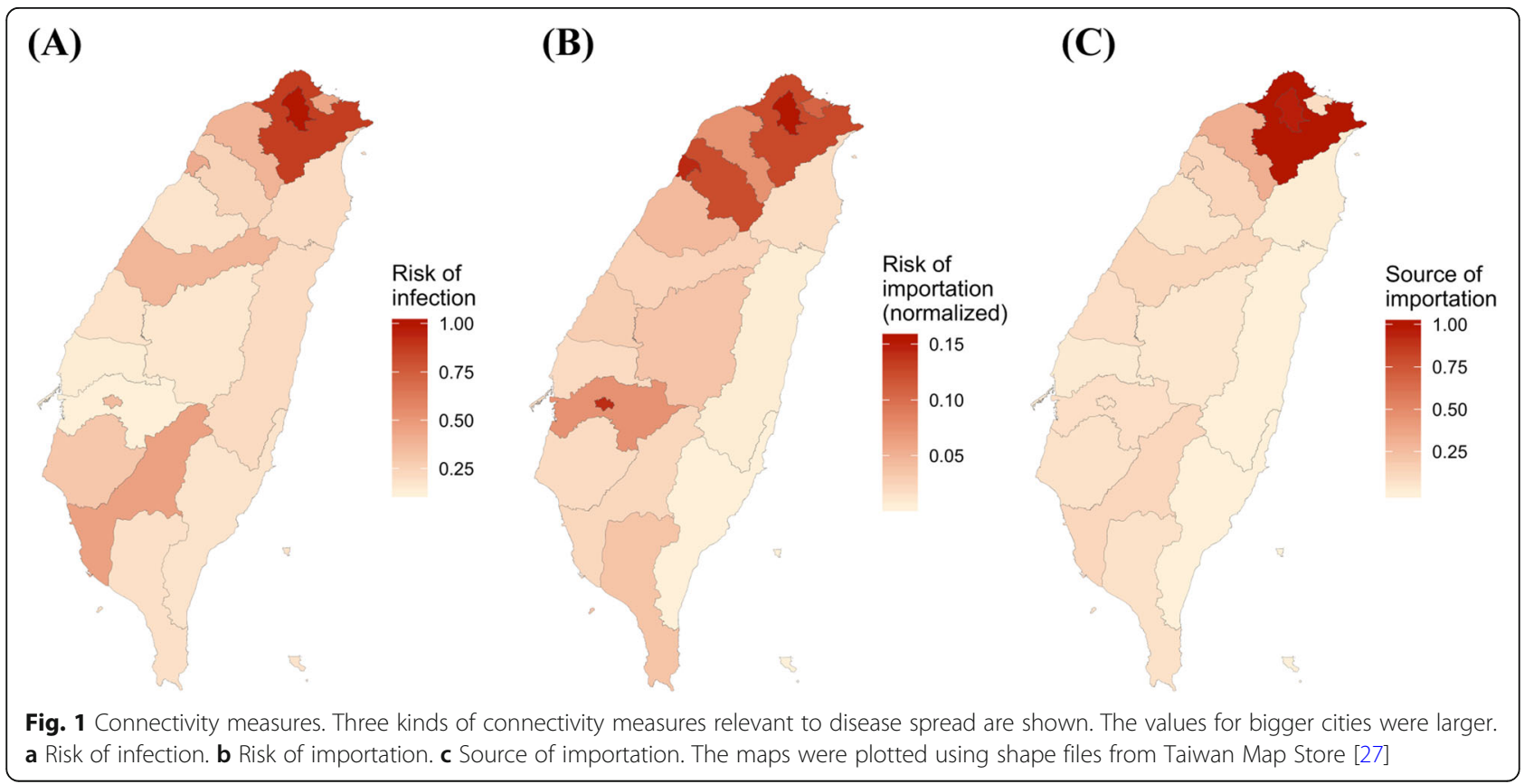


including both the number of initial infections and their locations. We developed a web-based interface (https:// roachchang.shinyapps.io/TW_CoV_Dynamics/) to show the geographic distribution of infections given different initial conditions, which can be readily used to inform targeted surveillance and control if SARS-CoV-2 starts spreading locally in Taiwan. Because other diseaserelevant hygiene behaviors, such as hand washing, mask wearing, or social distancing, may also have changed due to the awareness of COVID-19, we explored different transmission rates $\left(R_{O}=2.4,1.2\right.$, or 0.9$)$.

We considered different aspects of disease spread the probability of outbreak, the speed of spread, and the geographic range of outbreak. We estimated the probability of having more than 1000 infections (denoted by $P_{1000, k}$, where $k$ represents the number of initial infections) using stochastic simulations and used this to represent the probability of an outbreak. As expected, we found that, if we assumed that the transmission rates varied among cities (contact model), the probability of having more than 1000 infections also varied with the locations of initial infections, with the cities with larger risk of infection showing larger $P_{1000}$ (Figure S4A and Table S2). In simulations where 1000 infections were reached, the time it took to reach 1000 infections (denoted by $\left.T_{1000, k}\right)$ was also shorter for cities with larger risk of infection (Figure S4B). When assuming that the transmission rates in different cities were the same (residence model), the probability of having more than 1000 infections and the time to reach 1000 infections did not vary much with the locations of initial infections (Figure S5 and Table S3). The effect of intercity connectivity, however, was reflected in the variation in infection numbers across cities at $T_{1000}$ (denoted by $V_{1000}$ ). The variation in infection numbers was lower in cities with higher values of source of importation (Figure S4C) as the chance of spreading the virus to other cities was higher. In both models, well connected cities played more important roles, as they spread the virus to other cities more quickly and more widely.

\section{The impact of varying mobility on the risk of spread}

Above results were based on mobility data on regular days. Given that human mobility varied significantly in the past few months without travel restrictions imposed by the government, we further quantified the impact of varying mobility in Taiwan on the risk of spreading SARS-CoV-2. The impact was mainly reflected in the geographic range of infections in both models. When initial infections occurred in or around Lunar New Year, the speed of disease spread was enhanced (Fig. 2). Because mobility during Ching Ming Festival and Dragon Boat Festival differed less from regular days and these two holidays only lasted 4 days, it only led to minor differences in the geographic range of infections (Fig. 2). In the contact model, the probability of local outbreak was higher if initial infections occurred in or around Lunar New Year, and this impact was more apparent when initial infections were in locations with lower risk of infection (Figure S6).

\section{The effect of travel restrictions}

We examined the impact of varying mobility that occurred naturally during holidays on the disease spread above. Here we explored the level to which travel restrictions imposed by the government could potentially reduce the spread of SARS-CoV-2 in Taiwan at the initial stage of an outbreak. In both the contact model and the residence model, decreasing intracity movement (e.g. through quarantines or social distancing policies) had a much larger impact on $P_{1000,3}$ (Fig. 3 and Figure S11) and $T_{1000,3}$ (Figure S7) than decreasing intercity movement. The impact of reducing intercity travel was most evident in influencing how widespread the virus was: the infections were located in only a few cities at $T_{1000,3}$ if intercity travel was reduced (Figure S8).

We then investigated the impact of duration and timing of travel reductions (Fig. 4, and details at https:// roachchang.shinyapps.io/TW_CoV_Dynamics/). The probability of local outbreak decreased with increased duration of intracity travel reduction, but not change with the duration of intercity travel reduction. The results suggest that higher levels of reduction and longer periods of reduction for intracity travel can have similar impacts. For example, a $60 \%$ intracity travel reduction for 20 days had similar outcomes as a $70 \%$ reduction for 10 days. While $P_{1000,3}$ did not change with the length of intercity travel reduction, longer intercity travel reduction led to slower progression of the outbreak (higher $T_{1000,3}$ ) in the contact model and more clustered infections (higher $V_{1000,3}$ ) in both models (Figure S9). Furthermore, among the parameters we used, it was the best to reduce travel as early as possible to reduce the risk of outbreak (Figure S10).

\section{Discussion}

By utilizing aggregated human mobility data from Facebook, we characterized how mobility patterns in Taiwan changed since the emergence of COVID-19, and built metapopulation models to understand the potential spread of SARS-CoV-2 in Taiwan and to assess the potential impact of travel restrictions. We identified the top cities with the highest risk of infection as well as the top cities with the highest importation risk from other cities based on Facebook data and population sizes. We made a web-based interface showing the geographic distribution of infections at different time points $\left(T_{100}, T_{500}\right.$ and $\left.T_{1000}\right)$ in the 


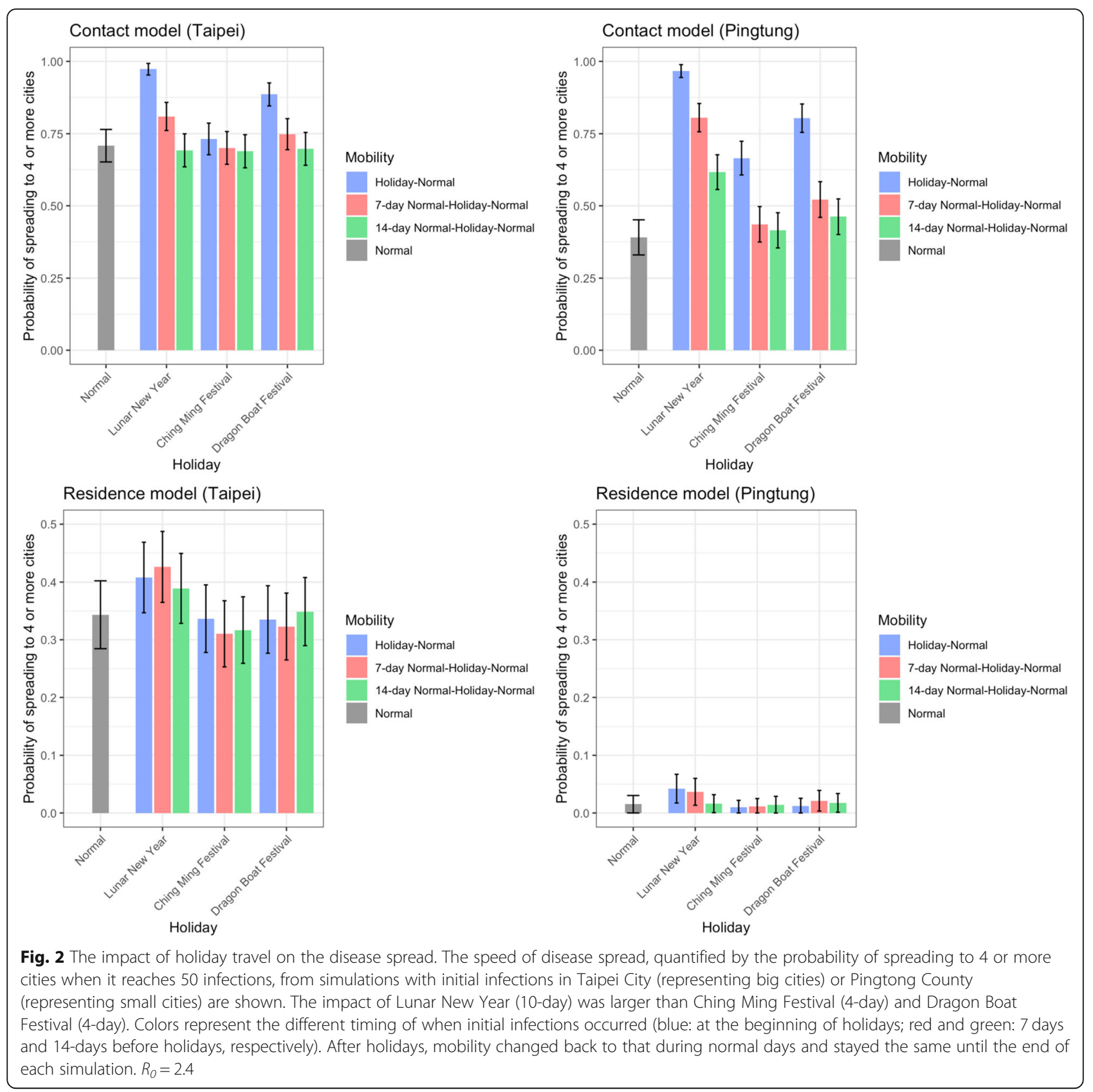

initial stages of the outbreak given different locations of initial infections. We demonstrate that these modeling results based on empirical mobility data can be obtained before an outbreak occurs, and can be readily used to help the public avoid high-risk areas, help public health professionals identify surveillance targets, and inform decisions on travel restrictions, providing one of the key elements for COVID-19 preparedness.

Consistent with previous findings showing that international or domestic travel bans are less effective than social distancing $[6,28]$, we found that intracity travel reduction has a higher impact on disease dynamics than intercity travel reduction, and increasing the length of intracity travel reduction increases the impact. Intercity travel reduction, however, influences the variation in infection numbers across cities and can reduce the number of cities that have infections at the initial stage of the outbreak. While intercity travel did not decrease the probability of outbreak, containing the infections to a few cities has important public health impacts, as this means surveillance 


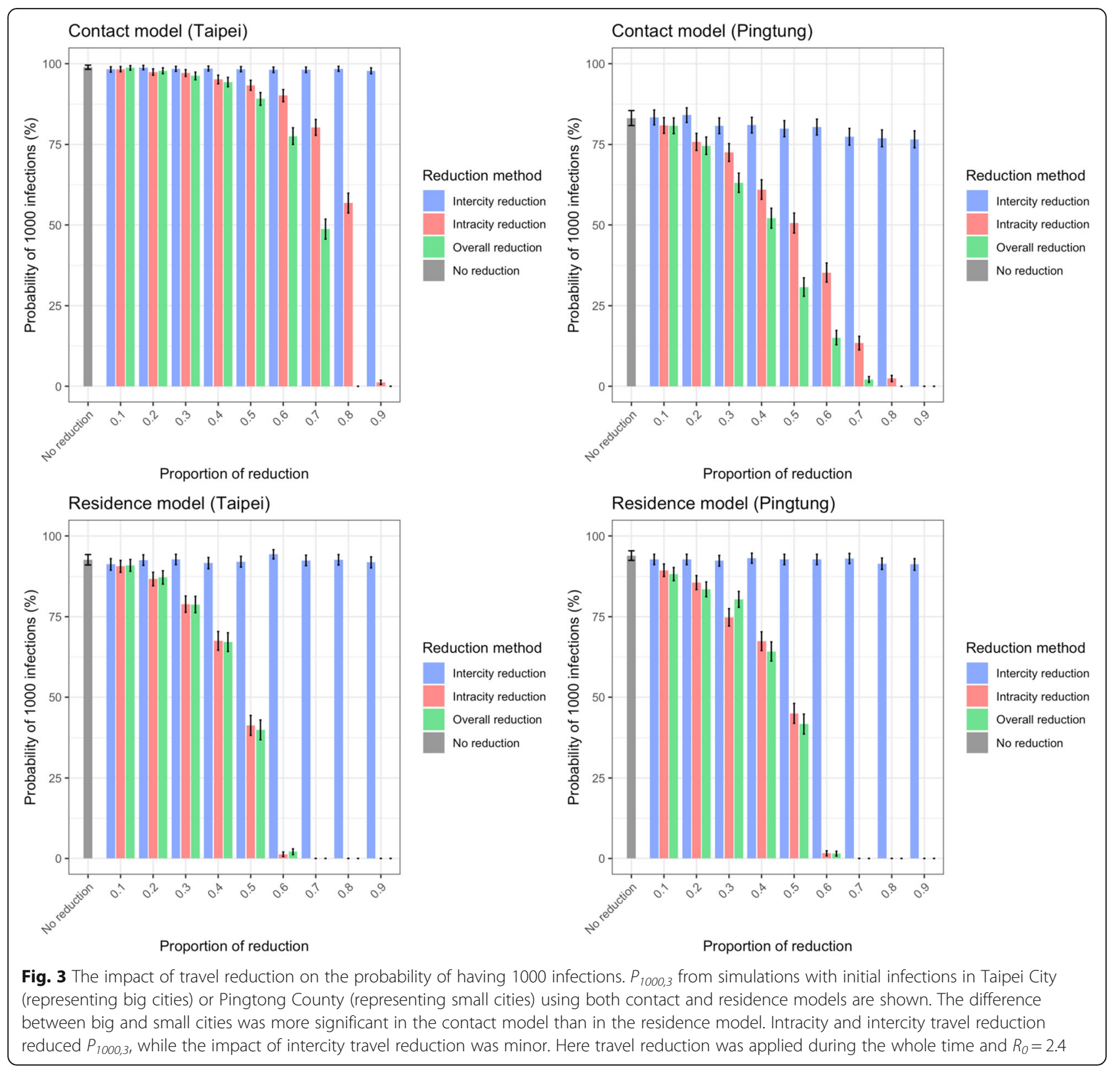

system can focus on fewer cities and control efforts can be more targeted. Our findings therefore suggest that once a case is identified, restriction of unnecessary intercity travel, can be important to geographically contain the spread in Taiwan.

Once a city experiences an outbreak, restrictions on intracity travel must be implemented. Intracity travel reduction in our model is effectively the same as any measure that can reduce contact rates between individuals, such as social distancing, or transmission probability given contact, such as hand washing or wearing facemasks. These measures have been shown to be effective in reducing the transmission of respiratory viral pathogens in both modeling and empirical studies [29-33], and should be encouraged. It has been shown that contact rates can be reduced by more than $70 \%$ during a lockdown [34].

Our study found that similar probabilities of an outbreak can occur with various combinations of length, level, and timing of travel reductions. Health officials can therefore take into consideration feasibility of different interventions, impact on society [35], and the capacity of the healthcare system to determine the optimal interventions and their duration [5]. Because the volume of travel in and around holidays can increase the speed of virus spread, our results suggest that it is important to avoid travel or reduce the impact of travel through 


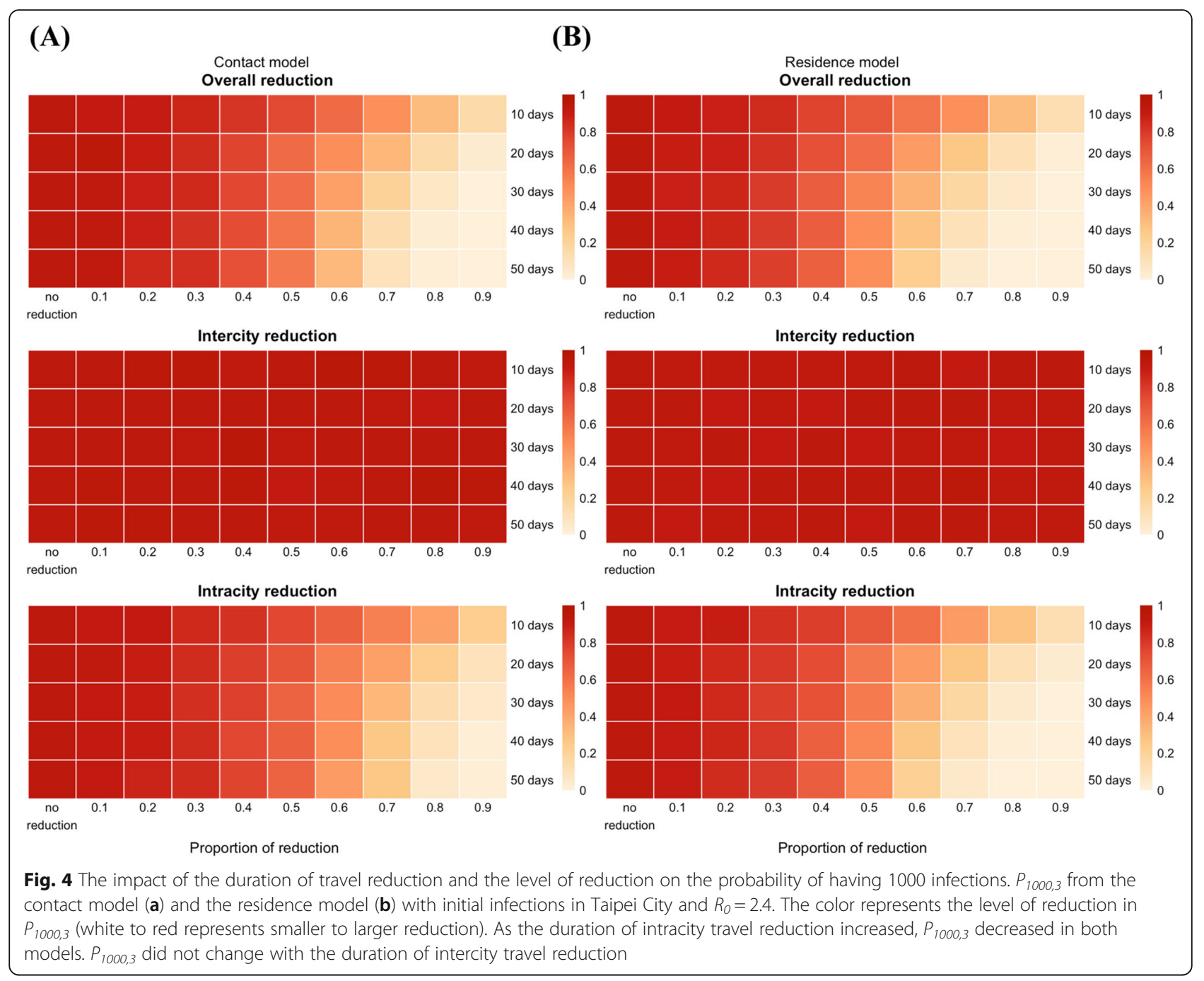

measures such as limiting social interactions and wearing facemasks when taking public transportation to reduce the spread of the virus.

Previous studies have shown that the impact of mobility or travel restrictions on infectious disease spread depends on region-specific mobility patterns, suggesting the importance of using empirical mobility data in modeling [36, 37]. We showed that Facebook mobility data can be used to track how the volume and pattern of travel change through time as the outbreak progresses, and we can incorporate any change in human mobility into the metapopulation models in nearly real time to help fight COVID-19 [12, 38]. While the Facebook data are limited to the population of Facebook users with location services turned on, these movement data can provide important insights into mobility patterns [18]. Additionally, Facebook coverage was consistent across cities (Table S4), suggesting minimal bias due to differential coverage by location. Moreover, our model utilizing human mobility data from Facebook is not limited to intercity or intracity level, or Taiwan. Facebook mobility data are also calculated at finer geographic scales (such as towns) and for other countries, and our model can be easily applied in these settings to understand disease dynamics of COVID-19.

\section{Conclusions}

In Taiwan, most cases to date were imported or linked to imported cases. To prepare for the potential spread within Taiwan, we utilized Facebook's aggregated and anonymized movement and colocation data to identify cities with higher risk of infection and regional importation. We showed that both intracity and intercity movement affect outbreak dynamics, with the former having more of an impact on the total numbers of cases and the latter impacting geographic scope. The timing, duration, and level of travel reduction together determine the impact of travel reductions on the number of infections, and multiple combinations of these 
can result in similar impact. These findings have important implications for guiding future policies for travel restrictions during outbreaks in Taiwan.

\section{Supplementary Information}

The online version contains supplementary material available at https://doi. org/10.1186/s12889-021-10260-7.

Additional file 1. Supplementary materials.

\section{Abbreviations}

COVID-19: Coronavirus Disease 2019; SLIR: Susceptible-latent-infectiousrecovered

\section{Acknowledgements}

We thank Brian Karrer, Alex Pompe, and Laura McGorman from Facebook This study was supported by the Ministry of Science and Technology in Taiwan (MOST 109-2636-B-007-006). MCC, YAL, and HHC were supported by Yushan Scholar Program, CSL was supported by the Ministry of Science and Technology in Taiwan (MOST109-2636-B-007-004), RK was supported by National Institute of General Medical Sciences (U54GM088558), COB was supported by National Institute of General Medical Sciences (R35GM124715-02). The funders had no role in study design, data collection, data analysis, data interpretation, or writing of the report.

\section{Authors' contributions}

$\mathrm{HHC}, \mathrm{CSL}$, and $\mathrm{COB}$ designed the experiments. $\mathrm{HHC}$ and RK created the models. MCC ran the simulations. MCC and HHC performed analysis. HHC, RK, and MCC interpreted the results and wrote the manuscript. YAL and CSL collected data for the models. All authors have read and approved the manuscript.

\section{Funding}

This study was supported by the Ministry of Science and Technology in Taiwan (MOST 109-2636-B-007-006). MCC, YAL, and HHC were supported by Yushan Scholar Program, CSL was supported by the Ministry of Science and Technology in Taiwan (MOST109-2636-B-007-004), RK was supported by National Institute of General Medical Sciences (U54GM088558), COB was supported by National Institute of General Medical Sciences (R35GM12471502). The funders had no role in study design, data collection, data analysis, data interpretation, or writing of the report. The content is solely the responsibility of the authors and does not necessarily represent the official views of the funders.

\section{Availability of data and materials}

Modeling output data are in the manuscript or can be accessed through publicly available web-based interface (https://roachchang.shinyapps.io/TW_ CoV_Dynamics/). Due to the Data Sharing Agreement with Facebook, readers can not access the original Facebook mobility data used in this study.

\section{Ethics approval and consent to participate}

This study received ethical approval from Research Ethics Committee of National Tsing Hua University (REC \#: 10812HM119). Aggregated, anonymized data were analyzed, and no individuals were enrolled into this study. Written permission was granted by Facebook for this study to access this data.

\section{Consent for publication}

Not applicable.

\section{Competing interests}

The authors declare that they have no competing interests.

\section{Author details}

'Department of Life Science \& Institute of Bioinformatics and Structural Biology, National Tsing Hua University, Hsinchu, Taiwan. ²Department of Epidemiology \& the Center for Communicable Disease Dynamics, Harvard T.H. Chan School of Public Health, Boston, MA, USA. ${ }^{3}$ Department of Life
Science \& Institute of Molecular and Cellular Biology, National Tsing Hua University, Hsinchu, Taiwan.

Received: 3 August 2020 Accepted: 17 January 2021

Published online: 27 January 2021

\section{References}

1. Dong E, Du H, Gardner L. An interactive web-based dashboard to track COVID-19 in real time. Lancet Infect Dis. 2020:20(5):533-4.

2. Maxmen A. More than 80 clinical trials launch to test coronavirus treatments. Nature. 2020;578(7795):347.

3. STAT. Covid-19 Drugs \& Vaccine Tracker [Internet]: STAT News; 2020. Available from: https://www.statnews.com/feature/coronavirus/drugsvaccines-tracker/

4. Ferguson NM, Laydon D, Nedjati-gilani G, Imai N, Ainslie K, Baguelin M, et al Impact of non-pharmaceutical interventions ( NPIs ) to reduce COVID- 19 mortality and healthcare demand. 2020; (March).

5. Kissler SM, Tedijanto C, Goldstein E, Grad YH, Lipsitch M. Projecting the transmission dynamics of SARS-CoV-2 through the postpandemic period. Science (80- ). 2020;368(6493):860 LP-868 Available from: http://science. sciencemag.org/content/368/6493/860.abstract.

6. Chinazzi M, Davis JT, Ajelli M, Gioannini C, Litvinova M, Merler S, et al. The effect of travel restrictions on the spread of the 2019 novel coronavirus (COVID-19) outbreak. Science. 2020;368(6489):395-400.

7. Bhatia S, Imai N, Cuomo-dannenburg G, Baguelin M, Boonyasiri A, Cori A, et al. Report 6: Relative sensitivity of international surveillance; 2020;(Figure 1). p. 1-6.

8. Niehus R, De Salazar PM, Taylor AR, Lipsitch M. Using observational data to quantify bias of traveller-derived COVID-19 prevalence estimates in Wuhan. China Lancet Infect Dis. 2020;20(7):803-8.

9. Taiwan CDC. COVID-19.

10. Bureau of Consular Affairs, Ministry of Foreign Affairs R of C (Taiwan). News \& Events [Internet]. Available from: https://www.boca.gov.tw/lp-220-2.html.

11. World Health Organization. Responding to community spread of COVID-19; 2020;(March). p. 1-6.

12. Buckee CO, Balsari S, Chan J, Crosas M, Dominici F, Gasser U, et al. Aggregated mobility data could help fight COVID-19. Science (80- ). 2020: eabb8021 Available from: http://science.sciencemag.org/content/early/2020/ 03/20/science.abb8021.abstract.

13. Li R, Pei S, Chen B, Song Y, Zhang T, Yang W, et al. Substantial undocumented infection facilitates the rapid dissemination of novel coronavirus (SARS-CoV2). Science (80- ). 2020:eabb3221 Available from: http://science.sciencemag.org/content/early/2020/03/13/science.abb3221. abstract.

14. Kraemer MUG, Yang C-H, Gutierrez B, Wu C-H, Klein B, Pigott DM, et al. The effect of human mobility and control measures on the COVID-19 epidemic in China. Science (80- ). 2020:eabb4218 Available from: http://science. sciencemag.org/content/early/2020/03/25/science.abb4218.abstract.

15. Gatto M, Bertuzzo E, Mari L, Miccoli S, Carraro L, Casagrandi R, et al. Spread and dynamics of the COVID-19 epidemic in Italy: Effects of emergency containment measures. Proc Natl Acad Sci. 2020;117(19):10484 LP-10491 Available from: http://www.pnas.org/content/117/19/10484.abstract.

16. Kissler S, Kishore N, Prabhu M, Goffman D, Beilin Y, Landau R, et al. Reductions in commuting mobility predict geographic differences in SARSCoV-2 prevalence in new York City. 2020;

17. Kishore N, Kiang MV, Engø-Monsen K, Vembar N, Schroeder A, Balsari S, et al. Measuring mobility to monitor travel and physical distancing interventions: a common framework for mobile phone data analysis. Lancet Digit Health. 2020:2(11):e622-8.

18. Maas P, Nayak C, Dow A, Gros A, Mason W, Filiz IO, et al. Facebook Disaster Maps: Methodology. Faceb Res. 2017:1-10 Available from: https://research. fb.com/facebook-disaster-maps-methodology/.

19. Allen LJS. A primer on stochastic epidemic models: formulation, numerical simulation, and analysis. Infect Dis Model. 2017;2(2):128-42.

20. Davies NG, Kucharski AJ, Eggo RM, Gimma A, Edmunds WJ, Jombart T, et al Effects of non-pharmaceutical interventions on COVID-19 cases, deaths, and demand for hospital services in the UK: a modelling study. Lancet Public Health. 2020;5(7):e375-85.

21. Ferguson N, Laydon D, Nedjati Gilani G, Imai N, Ainslie K, Baguelin M, et al. Report 9: impact of non-pharmaceutical interventions (NPIs) to reduce COVID19 mortality and healthcare demand. 2020; 
22. Ruktanonchai NW, DeLeenheer $\mathrm{P}$, Tatem AJ, Alegana VA, Caughlin TT, zu Erbach-Schoenberg E, et al. Identifying Malaria Transmission Foci for Elimination Using Human Mobility Data. PLoS Comput Biol. 2016;12(4): e1004846 Available from: https://doi.org/10.1371/journal.pcbi.1004846.

23. Acevedo MA, Prosper O, Lopiano K, Ruktanonchai N, Caughlin TT, Martcheva M, et al. Spatial heterogeneity, host movement and mosquitoborne disease transmission. PLoS One. 2015;10(6):e0127552 Available from: https://pubmed.ncbi.nlm.nih.gov/26030769.

24. Cosner C, Beier JC, Cantrell RS, Impoinvil D, Kapitanski L, Potts MD, et al. The effects of human movement on the persistence of vector-borne diseases. J Theor Biol. 2009;258(4):550-60 2009/03/03. Available from: https://pubmed. ncbi.nlm.nih.gov/19265711.

25. Rodríguez DJ, Torres-Sorando L. Models of infectious diseases in spatially heterogeneous environments. Bull Math Biol. 2001;63(3):547-71 Available from: https://doi.org/10.1006/bulm.2001.0231.

26. McCallum H, Barlow N, Hone J. How should pathogen transmission be modelled? Trends Ecol Evol. 2001;16(6):295-300.

27. National Land Surveying and Mapping Center. Taiwan Map Store. https:// whgis.nlsc.gov.tw/English/5-1Files.aspx.

28. Wu JT, Leung K, Leung GM. Nowcasting and forecasting the potential domestic and international spread of the 2019-nCoV outbreak originating in Wuhan, China: a modelling study. Lancet. 2020;395(10225):689-97.

29. Lai S, Ruktanonchai NW, Zhou L, Prosper O, Luo W, Floyd JR, et al. Effect of non-pharmaceutical interventions to contain COVID-19 in China. Nature. 2020; Available from: https://doi.org/10.1038/s41586-020-2293-x.

30. Prem K, Liu Y, Russell TW, Kucharski AJ, Eggo RM, Davies N, et al. The effect of control strategies to reduce social mixing on outcomes of the COVID-19 epidemic in Wuhan, China: a modelling study. Lancet Public Health. 2020: 5(5):E261-70.

31. Anderson RM, Heesterbeek H, Klinkenberg D, Hollingsworth TD. How will country-based mitigation measures influence the course of the COVID-19 epidemic? Lancet. 2020;395(10228):931-4.

32. Cowling BJ, Chan K-H, Fang VJ, Cheng CKY, Fung ROP, Wai W, et al. Facemasks and hand hygiene to prevent influenza transmission in households: a cluster randomized trial. Ann Intern Med. 2009;151(7):437-46.

33. Tracht SM, Del Valle SY, Hyman JM. Mathematical Modeling of the Effectiveness of Facemasks in Reducing the Spread of Novel Influenza A (H1N1). PLoS One. 2010;5(2):e9018 Available from: https://doi.org/10.1371/ journal.pone.0009018.

34. Jarvis Cl, Van Zandvoort K, Gimma A, Prem K, Klepac P, Rubin GJ, Edmunds WJ. Quantifying the impact of physical distance measures on the transmission of COVID-19 in the UK. BMC Med. 2020;18:1-10.

35. Massaro E, Ganin A, Perra N, Linkov I, Vespignani A. Resilience management during large-scale epidemic outbreaks. Sci Rep. 2018;8(1):1-9.

36. Gómez-Gardenes J, Soriano-Panos D, Arenas A. Critical regimes driven by recurrent mobility patterns of reaction-diffusion processes in networks. Nat Phys. 2018;14(4):391-5

37. Dalziel BD, Pourbohloul B, Ellner SP. Human mobility patterns predict divergent epidemic dynamics among cities. Proc R Soc B Biol Sci. 2013; 280(1766):20130763.

38. Emanuele Pepe, Paolo Bajardi, Laetitia Gauvin, Filippo Privitera, Ciro Cattuto MT. COVID-19 outbreak response: first assessment of mobility changes in Italy following lockdown [Internet]. 2020. Available from: https://covid19mm. github.io/in-progress/2020/03/13/first-report-assessment.html

\section{Publisher's Note}

Springer Nature remains neutral with regard to jurisdictional claims in published maps and institutional affiliations.

Ready to submit your research? Choose BMC and benefit from:
- fast, convenient online submission
- thorough peer review by experienced researchers in your field
- rapid publication on acceptance
- support for research data, including large and complex data types
- gold Open Access which fosters wider collaboration and increased citations
- maximum visibility for your research: over 100M website views per year
At BMC, research is always in progress.
Learn more biomedcentral.com/submissions

